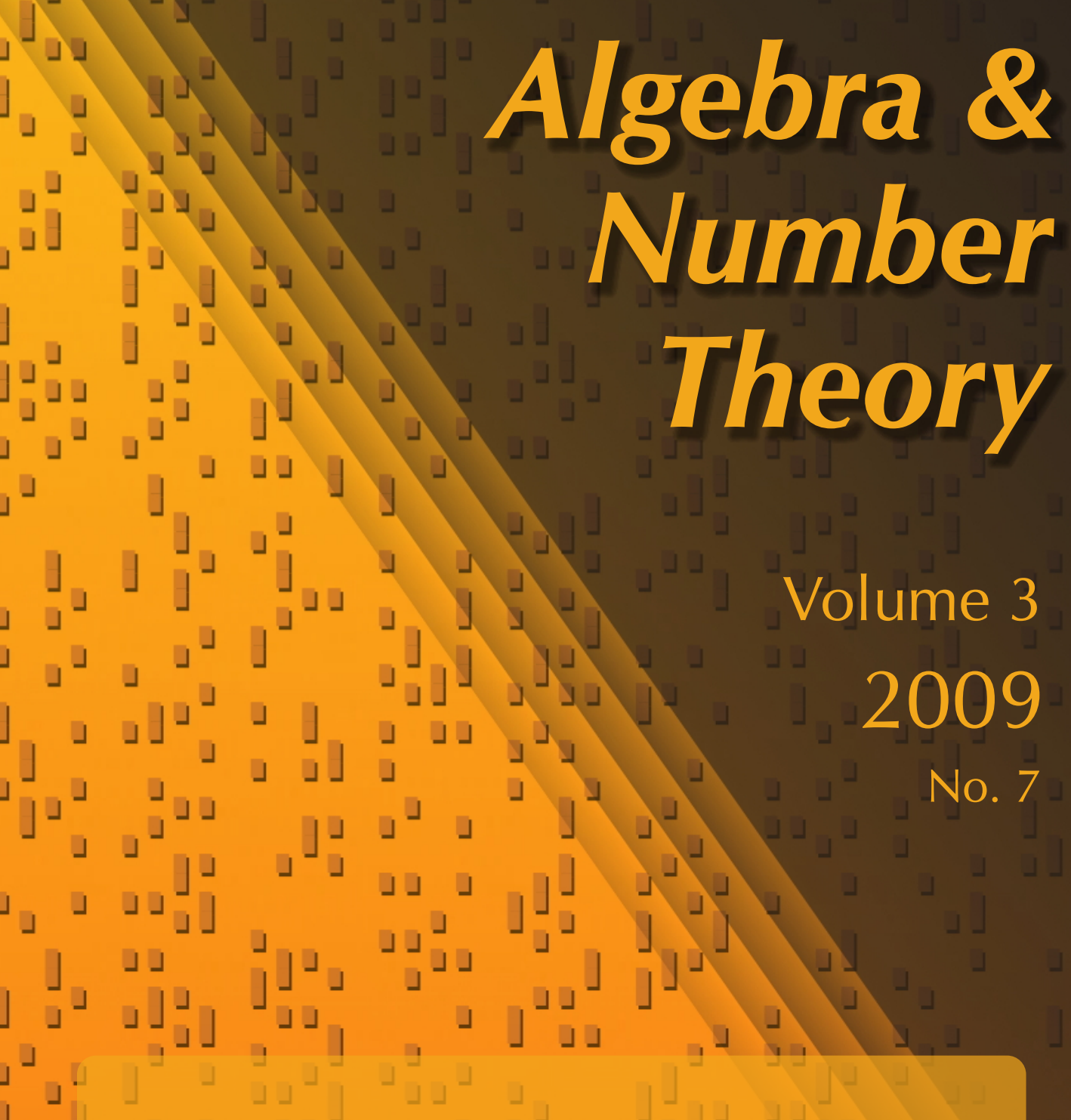

\title{
Discretely ordered groups
}

Peter A Linnell, Akbar Rhemtulla and Dale P. O. Rolfsen

\lrcorner

\lrcorner

\lrcorner
\lrcorner

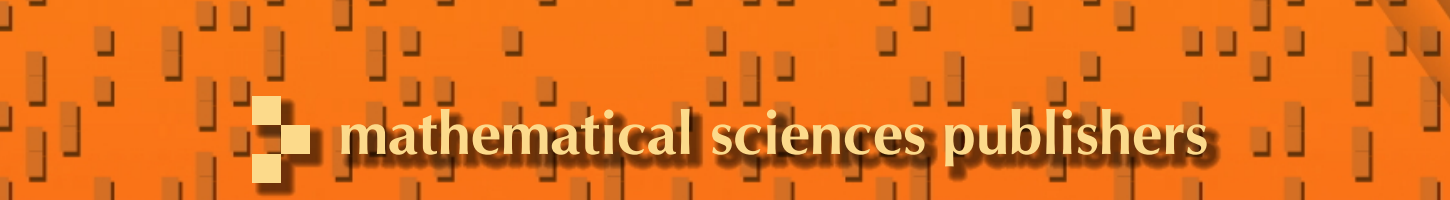




\title{
Discretely ordered groups
}

\author{
Peter A Linnell, Akbar Rhemtulla and Dale P. O. Rolfsen
}

\begin{abstract}
We consider group orders and right-orders which are discrete, meaning there is a least element which is greater than the identity. We note that nonabelian free groups cannot be given discrete orders, although they do have right-orders which are discrete. More generally, we give necessary and sufficient conditions that a given orderable group can be endowed with a discrete order. In particular, every orderable group $G$ embeds in a discretely orderable group. We also consider conditions on right-orderable groups to be discretely right-orderable. Finally, we discuss a number of illustrative examples involving discrete orderability, including the Artin braid groups and Bergman's nonlocally-indicable right orderable groups.
\end{abstract}

\section{Introduction}

Let $G$ be a group and suppose < is a strict total order relation on the set of its elements. Then $(G,<)$ is a right-ordered group if $f<g$ implies that $f h<g h$ for all $f, g, h \in G$. If in addition $f<g$ implies that $h f<h g$, then we say $(G,<)$ is an ordered group. If such an order exists for a given group $G$, we say that $G$ is right-orderable or orderable, respectively. We call the order $<$ discrete if there is an element $a \in G$ such that $1<a$, where 1 denotes the identity element of $G$, and there is no element of $G$ strictly between these.

For a right-ordered group, the positive cone $P:=\{g \in G \mid 1<g\}$ satisfies

(1) $P$ is closed under multiplication and

(2) for every $g \in G$, exactly one of $g=1, g \in P$ or $g^{-1} \in P$ holds.

Conversely, if a group $G$ has a subset $P$ with properties (1) and (2), it is routine to verify that the order defined by $g<h$ if and only if $h g^{-1} \in P$ makes $(G,<)$ a right-ordered group. Similarly, a group $G$ is orderable if and only if it admits a subset $P$ satisfying (1), (2) and

(3) $g P g^{-1}=P$ for all $g \in G$.

MSC2000: primary 20F60; secondary 06F15, $20 \mathrm{~F} 36$.

Keywords: discrete order. 
A subset $X$ of a (right-) ordered group $G$ is convex if $x<y<z$ and $x, z \in X$ imply $y \in X$. We recall that the set of all convex subgroups of a (right-) ordered group is linearly ordered by inclusion. A convex jump $C \longmapsto D$ is a pair of distinct convex subgroups such that $C \subset D$ and there are no convex subgroups strictly between them. In particular, the convex jump determined by a nonidentity element $g$ of $G$ is defined by $C=$ the union of all convex subgroups not containing $g$ and $D=$ the intersection of all convex subgroups which do contain $g$. If the group is orderable, then for any convex jump $C \longmapsto D, C$ is normal in $D$ and the quotient $D / C$ embeds in $\mathbb{R}$, the additive reals, by an order-preserving isomorphism.

Lemma 1.1. If $<$ is a discrete right-order on $G$ and $a$ is the least positive element under $<$, then $\langle a\rangle$ is convex. Moreover, for any $g \in G$, we have $a^{-1} g<g<a g$ and there is no element strictly between these elements of $G$. If the right-order $<$ is not discrete, then it is dense in the sense that for any $f, g \in G$ with $f<g$, there exists $h \in G$ with $f<h<g$.

Proof. Since $a^{-1}<1<a$, we see that $a^{-1} g<g<a g$. If $g<x<a g$, then $1<x g^{-1}<a$, a contradiction. Hence there is no element strictly between $g$ and $a g$. Similarly there is no element between $a^{-1} g$ and $g$. In particular, for any integers $n<m, a^{n}<g<a^{m}$ implies $g \in\langle a\rangle$ and thus $\langle a\rangle$ is convex. If there exists $f<g$ with no element strictly between, a routine calculation shows $g f^{-1}$ is the least positive element for the order.

Note that according to our definitions, the trivial group 1 has exactly one right order, and this order is dense but not discrete.

Situated strictly between the class of right-orderable groups and the class of orderable groups is the class of locally indicable groups. Recall that a group $G$ is locally indicable if every finitely generated nontrivial subgroup of $G$ has an infinite cyclic quotient. Such groups are right-orderable, as was shown by Burns and Hale [1972]. On the other hand, a right-orderable group need not be locally indicable as was shown by Bergman [1991]. However for a large class of groups the class of right-orderable groups coincides with the class of locally indicable groups. Further results on this topic are contained in [Linnell 2001], [Longobardi et al. 2000], and especially in [Morris 2006].

Our interest in considering locally indicable groups $G$ is due to the fact that such groups have a series (defined below) with torsion-free abelian factors as shown in [Brodskiı 1984]. They possess a right-order in which the set of convex subgroups form a series with factors which are order isomorphic to subgroups of the additive group of reals; we shall refer to such orders as lexicographic. (Such orders are also called Conrad orders and are characterized by the condition that if $g>1$ and $h>1$, then there exists a positive integer $n$ such that $(g h)^{n}>h g$; see [Botto Mura and Rhemtulla 1977, §7.4] for further details.) Note that for such an ordering, any 
nontrivial element $g \in G$ is positive if and only if the cosets satisfy $C g>C$ in the factor group $D / C$ determined by $g$.

By a series for $G$ we mean a set $\Sigma=\left\{H_{\lambda} \mid \lambda \in \Lambda\right\}$ of subgroups of $G$, where $\Lambda$ is a totally ordered set of indices, satisfying:

- if $\lambda<\mu$ then $H_{\lambda} \subset H_{\mu}$,

- $\{1\}$ and $G$ belong to $\Sigma$,

- $\Sigma$ is closed under arbitrary unions and intersections,

- if $\mu$ immediately follows $\lambda$ in $\Lambda$, then $H_{\lambda}$ is normal in $H_{\mu}$ and $H_{\mu} / H_{\lambda}$ is called a factor associated to the jump $H_{\lambda} \longmapsto H_{\mu}$.

In the next section we characterize groups that have discrete orders. We show that a group $G$ has a discrete order if and only if it is an orderable group and its center $Z(G)$ contains an isolated infinite cyclic group. Recall that a subgroup $H$ of a group $G$ is said to be isolated if $g \in G$ and $g^{n} \in H$ for some $n>0$ implies $g \in H$.

In Section 3, we deal with groups possessing discrete lexicographic right-orders and discrete right-orders. It will follow, in particular, that any nontrivial finitely generated orderable group has discrete right-orders and if it has a central order (as is the case for free groups, pure braid groups and wreath products or free products of such groups), then it has discrete lexicographic right-orders. Recall that an order $<$ on $G$ is called central if for every convex jump $C \longmapsto D$, we have $[D, G] \subseteq C$ where $[D, G]$ denotes the subgroup $\left\langle d^{-1} g^{-1} d g \mid d \in D, g \in G\right\rangle$.

The result is of course not true for orderable groups in general. The additive group of rational numbers has no discrete right-order.

The final section presents examples of discretely ordered groups which have nontrivial subgroups (for example, the commutator subgroup) upon which the restriction of the given order is dense. We also note that there exist finitely generated right-orderable groups, for example, the Artin braid groups $B_{n}, n \geq 5$, that are not locally indicable, yet have a discrete right-order.

\section{Discrete orders}

Theorem 2.1. If $<$ is a discrete order on a group $G$, then there exists an element $z$ in the center $Z(G)$ such that $\langle z\rangle$ is convex under $\langle$ and $1 \longmapsto\langle z\rangle$ is a jump. Conversely, if $G$ is an orderable group and $Z(G)$ contains an isolated infinite cyclic group, then there is a discrete order on $G$.

Proof. Let $<$ be a discrete order on $G$ with $z>1$ as the minimal positive element. Then $g^{-1} z g$ is positive for every $g \in G$. Moreover, $z<g^{-1} z g$ implies $1<g z g^{-1}<z$, a contradiction. Thus $z \in Z(G)$. Also Lemma 1.1 shows that $\langle z\rangle$ is convex under $<$ and $1 \longmapsto\langle z\rangle$ is a jump. 
Conversely, let $\langle z\rangle$ be an isolated subgroup in the center $Z(G)$ of an orderable group $G$. Since $G$ is orderable, so is $G / Z(G)$; see [Botto Mura and Rhemtulla 1977, Theorem 2.2.4]. Moreover, $Z(G) /\langle z\rangle$ is orderable since $\langle z\rangle$ is isolated in $Z(G)$. Order $\langle z\rangle$ (with $z$ positive), $Z(G) /\langle z\rangle$ and $G / Z(G)$. Now order $G$ as follows. If $1 \neq g \in G \backslash Z(G)$, then put $g$ in the positive cone if $g Z(G)$ is positive; if $g \in Z(G) \backslash\langle z\rangle$, then put $g$ in the positive cone if $g\langle z\rangle$ is positive; if $g=z^{n}$, then put $g$ in the positive cone if $0<n$. It is routine to verify that this gives a discrete order on $G$ with $z$ as the minimal positive element.

Corollary 2.2. For any orderable group $G$, the group $\mathbb{Z} \times G$ has a discrete order. In particular, every orderable group embeds in a discretely orderable group, whose order extends the given order.

\section{Discrete right orders}

We begin this section with the following result which is easy to prove. It is not required in the proofs of the other results.

Lemma 3.1. If $(G,<)$ is a nontrivial right-ordered group such that the order $<i s$ a well order on the set of positive elements of $G$, then $G$ is infinite cyclic.

Lemma 3.2. If $<$ is a discrete right order on $G$ and a the least positive element under $<$ then for any element $1<g \in G$, we have $1<a g a^{-1}$ and $1<a^{-1} g a$.

Proof. Since $a \leq g, 1 \leq g a^{-1}$. Thus $a g a^{-1}$ is a product of two positive elements and hence positive. By Lemma 1.1, there is no element of $G$ strictly between $a^{-1} g$ and $g$. Since $1<g, 1 \leq a^{-1} g$ and so $a \leq a^{-1} g a$.

Definition 3.3. Let $<$ be a right order on a group $G, C$ a subgroup of $G$ and $a \in G$. We shall say that conjugation by a preserves order on $C$ to mean that $C$ is normalized by $\langle a\rangle$ and conjugation by $a$ and by $a^{-1}$ preserves the order on $(C,<)$.

Lemma 3.4. Suppose < is a right order on a group $G, C$ is a subgroup of $G$, $1 \neq a \in G$ and $C \cap\langle a\rangle=1$. If conjugation by a preserves order on $C$, then there is a discrete right order on the subgroup $\langle C, a\rangle$ with a as the minimal positive element. Moreover, this right order and the given right order agree on $C$. Finally if $a E a^{-1}=E$ for all convex subgroups $E$ of $C$, then the convex subgroups of $H$ under this new right order are $\{1\}$ and $\langle a, E\rangle$, where $E$ is a convex subgroup of $C$.

Proof. Set $H=\langle a, C\rangle$. An element $g \in H$ has a unique expression as $g=a^{n} c$ where $c \in C$ and $n \in \mathbb{Z}$. Define the set $P$ as follows: $g \in P$ if $1<c$ or $c=1$ and $n>0$. Note that $P \cup P^{-1}=H \backslash\{1\}$ and $P \cap P^{-1}=\varnothing$. Moreover if $g=a^{n} c$ and $h=a^{m} d$ are in $P$, then their product $g h=a^{n+m}\left(a^{-m} c a^{m}\right) d \in P$ as conjugation by $a^{m}$ preserves order on $C$. Thus the order $\prec$ on $H$ given by $g \prec h$ if and only if $h g^{-1} \in P$ is a right order on $H$. Furthermore if $g=a^{n} c$ and $h=a^{m} d$ are in $H$, 
then $g \prec h$ if and only if $c<d$ or $c=d$ and $n<m$. It is now clear that $a$ is the least positive element under this order, and that $<$ and $\prec$ agree on $C$. Finally we verify that the convex subgroups are $\langle a, E\rangle$, where $E$ is a convex subgroup of $C$.

Set $A=\langle a\rangle$, so $H=A C$. If $K$ is a nontrivial convex subgroup of $H$, then $a \in K$ and $C \cap K$ is a convex subgroup of $C$, and we have $(K \cap C) A=K \cap C A=K$. Thus $K=\langle a, E\rangle$ where $E=C \cap K$. On the other hand if $E$ is a convex subgroup of $C$, we claim that $A E$ is a convex subgroup of $H$. Suppose $a^{m} b \prec a^{n} c \prec a^{p} d \in E A$, where $b, d \in E, c \in C$, and $m, n, p \in \mathbb{Z}$. Then $b \leq c \leq d$ and hence $c \in E$, and it follows that $A E$ is a convex subgroup of $(H, \prec)$, as required.

Theorem 3.5. Let $(G,<)$ be an ordered group, $1 \neq a \in G$ and $C \longmapsto D$ the convex jump determined by a (thus $a \in D \backslash C$ and $D / C$ is torsion-free abelian). If $D /\langle a, C\rangle$ is torsion free, then there is a discrete right order on $G$ with a as the minimal positive element. Moreover, if also $[a, F] \subseteq E$ for every jump $E \longmapsto F$, then there is a discrete lexicographic right order on $G$ (with a as minimal positive element).

Proof. Set $H=\langle a, C\rangle$. The hypothesis of Lemma 3.4 applies and we right order $H$ as described there. Next we order the factor group $D / H$. This is possible since $D / H$ is torsion-free abelian. Define the set $Q \subset G$ as follows. If $g \in H$, then $g \in Q$ if $g$ is positive in the order on $H$ described above. If $g \in D \backslash H$, then put $g$ in $Q$ if $g H$ is positive in the order on $D / H$ given above. If $g \in G \backslash D$, then put $g$ in $Q$ if $g$ is positive in $(G,<)$, the original order on $G$.

It is routine to verify that $Q \cup Q^{-1}=G \backslash\{1\}, Q \cap Q^{-1}=\varnothing$ and $Q Q \subseteq Q$, thus giving a right order $\prec$ on $G$ with $Q=\{g \in G \mid 1 \prec g\}$.

The same right order $\prec$ is lexicographic if $[a, F] \subseteq E$ for every jump $E \longmapsto F \subseteq C$. The convex subgroups are $\{1\},\langle a\rangle$, and $\langle a, E\rangle$ for every subgroup $E$ convex under the original order $<$. This follows from Lemma 3.4: note that $E=\langle a, E\rangle$ if $E \geq D$ and $E \cap C$ is a convex subgroup of $C$.

Corollary 3.6. Nontrivial free groups have discrete lexicographic right orders.

Proof. This follows from the fact that the descending lower central series terminates in $\{1\}$ and the factors are free abelian groups. Thus any element may be made to be the least positive element so long as it is a primitive element of the factor group that is determined by the element.

Corollary 3.6 can be generalized to free partially commutative groups. These are described in [Duchamp and Krob 1992, §1.1], and the definition given there does not require these groups to be finitely generated. Free partially commutative groups are known under many other names, in particular they are also called right-angled Artin groups [Charney 2007], at least for finitely generated groups.

Corollary 3.7. Nontrivial free partially commutative groups have discrete lexicographic right orders. 
Proof. Free partially commutative groups are residually nilpotent by [Duchamp and Krob 1992, Theorem 2.3]. Furthermore [Duchamp and Krob 1992, Theorems $1.1,2.1]$ show that the quotients of the lower central series are free abelian groups. The result now follows from Theorem 3.5.

Note that a nonabelian free group does not have a discrete order. This follows from Theorem 2.1.

Finally, all surface groups (orientable or not) except the Klein bottle and projective plane are residually torsion-free nilpotent, by [Baumslag 1968, Theorem 1] (we would like to thank Warren Dicks for this reference). Thus these surface groups also have lexicographic discrete right orders. With the exception of the torus, these groups have trivial center and therefore do not enjoy discrete orders.

The pure braid groups $P_{n}$, like free groups and surface groups, are also residually torsion-free nilpotent. But, unlike those examples, the groups $P_{n}$ do have discrete orders. The center $Z\left(P_{n}\right)$ is infinite cyclic, generated by $z=$ the full twist braid (often denoted $\Delta_{n}^{2}$ ). Since $\langle z\rangle$ is trivially isolated in $Z\left(P_{n}\right)$, the second part of Theorem 2.1 provides a discrete order with $\Delta_{n}^{2}$ as least positive element. In fact any discrete order of $P_{n}$ must have $\Delta_{n}^{2}$ (or its inverse) as least positive element.

\section{Examples}

A group may have a lexicographic right order and not have any discrete right order even when the factors formed by the convex jumps are all infinite cyclic. One example of this is the following.

Example 4.1. Let $G=\left\langle a_{i} \mid i \in \mathbb{Z}\right\rangle$ with defining relations $\left[a_{i}, a_{j}\right]=1$ if $|i-j|>1$ and $a_{i+1} a_{i} a_{i+1}^{-1}=a_{i}^{-1}$.

Every right order on $G$ is lexicographic with the subgroups $\left\langle a_{i} \mid i<j\right\rangle$ forming the chain of convex subgroups, and every right order is determined by the $a_{i}$ (that is, whether or not $a_{i}$ is in the positive cone for each $i \in \mathbb{Z}$ ). This construction is just the expansion of the well known (Klein bottle) group $D=\langle a, b\rangle$ where $b^{-1} a b=a^{-1}$. There are exactly four right orders on $D$, every one discrete with $a$ or $a^{-1}$ as the minimal positive element.

We show next that any infinite cyclic extension of the group $G$ of this example has a discrete right order if it is finitely generated. However we can have a metacyclic extension of $G$ that is finitely generated and right orderable but without any discrete right order. These are given as Proposition 4.2 and Example 4.3. If $x, t$ are elements of a group, then $x^{t}$ will denote $t^{-1} x t$.

Proposition 4.2. Let $\Gamma=G\langle t\rangle$ be a finitely generated infinite cyclic extension of the group $G$ given in Example 4.1. Then $\Gamma$ has a discrete right order with $t$ (or $\left.t^{-1}\right)$ as the minimal positive element. 
Proof. Every nontrivial element $g \in G$ has unique expression of the form

$$
g=a_{r_{1}}^{d_{1}} \ldots a_{r_{k}}^{d_{k}}
$$

where $r_{1}<\cdots<r_{k}$ and $d_{i} \neq 0$ for all $i$. Call $a_{r_{k}}^{d_{k}}$ the leading term of $g$ and denote it by $\ell(g)$. Call $r_{k}$ the leading suffix of $g$.

Note that $\ell\left(g^{n}\right)=(\ell(g))^{n}$ for all $n \in \mathbb{Z} \backslash\{0\}$. Moreover, if $\ell(g)=a_{r}^{j}, \ell(h)=a_{s}^{k}$ and $r<s$, then $\ell(g h)=\ell(h g)=\ell(h)$. Since $\left(a_{i+1}^{t}\right)^{-1}\left(a_{i}^{t}\right)\left(a_{i+1}^{t}\right)=\left(a_{i}^{t}\right)^{-1}$, we see that the leading suffix of $\left(a_{i+1}\right)^{t}$ is greater than that of $\left(a_{i}\right)^{t}$ by at least one. Thus also the leading suffix of $\left(a_{i+1}\right)^{t^{-1}}$ is greater than that of $\left(a_{i}\right)^{t^{-1}}$ by at least one, and we deduce that the leading suffix of $\left(a_{i+1}\right)^{t}$ is greater than that of $\left(a_{i}\right)^{t}$ by exactly one. It follows that $i>j$ implies that the leading suffix of $\left(a_{i}\right)^{t}$ is greater than that of $\left(a_{j}\right)^{t}$ by exactly $i-j$.

Since $\Gamma$ is finitely generated, the leading suffix of $a_{i}^{t}$ (or that of $a_{i}^{t^{-1}}$ ) is greater than $i$ for at least one value of $i$ - otherwise $\left\langle a_{j} \mid j \leq i\right\rangle$ is normal in $\Gamma$ for every $i$, and hence $\Gamma$ can not be finitely generated. Thus if the leading suffix of $a_{0}^{t}$ is $n$, then the leading suffix of $a_{i}^{t}$ is $i+n$ for every integer $i$, and we may assume that $n>0$.

We now right order the group $G$ by putting $a_{0}, a_{1}, \ldots, a_{n-1}$ in the positive cone $P$. Next, for all $n \leq r<2 n$, we put $a_{r} \in P$ if the exponent of $\ell\left(a_{r-n}^{t}\right)$ is positive and $a_{r}^{-1} \in P$ otherwise. Next put $a_{r+n}$ or $a_{r+n}^{-1}$ in $P$ depending on whether the exponent of $\ell\left(\left(\ell\left(a_{r-n}\right)^{t}\right)^{t}\right)$ is positive or negative. Continue this process. For every integer $i \geq 0$ we have determined whether $a_{i}$ or its inverse is in $P$. Next, for $0>r \geq-n$ put $a_{r} \in P$ if the exponent of $\ell\left(a_{r+n}^{t^{-1}}\right)$ is positive. Put $a_{r}^{-1} \in P$ otherwise. Continue this process. This takes care of all $a_{i}$ for $i \in \mathbb{Z}$. Note that the above order on $G$ is $\langle t\rangle$ invariant. Hence by Lemma 3.4, $\Gamma$ has a right discrete order with $t$ as the minimal positive element.

Example 4.3. Let $G$ be the group in Example 4.1. Consider the map

$$
\phi:\left\{a_{i} \mid i \in \mathbb{Z}\right\} \rightarrow\left\{a_{i}^{-1} \mid i \in \mathbb{Z}\right\}
$$

given by $\phi\left(a_{i}\right)=a_{i}^{-1}$. Then $\phi$ extends uniquely to an automorphism of $G$ that inverts every $a_{i}$. Let $\langle G, u\rangle$ be the infinite cyclic extension of $G$ by $\langle u\rangle$ where $u^{-1} a_{i} u=a_{i}^{-1}$ for all $i \in \mathbb{Z}$. Note that $\langle G, u\rangle$ is right orderable because it is an infinite cyclic extension of the right orderable group $G$. However, it has no discrete right order. This can be seen as follows. Suppose $g \in G$ and $c:=g u^{j}$ is a minimal positive element under some right order $\langle$ on $\langle G, u\rangle$. Since $G$ has no discrete right order, $j \neq 0$ and it must be even otherwise $c^{-1} a_{i} c=a_{i}^{-1}$ for some $i$, contradicting Lemma 3.2.

Suppose $1<u$. Then $a_{i}^{r}<u$ for every $i, r \in \mathbb{Z}$, for if $1<u<a_{i}^{r}$, then $u a_{i}^{-r}<1$. Hence $a_{i}^{r}=u a_{i}^{-r} u^{-1}<1$, a contradiction. Thus $h<u$ for all $h \in G$. Since 
$1<c=g u^{j}=u^{j} g$, we see that $j$ is positive, and then we have $1<u^{j-1}, u g$, which contradicts the hypothesis that $c$ is the minimal positive element. The argument is similar if $u<1$. We note in particular that if $1<u$, then $h<u$ for every $h \in G$.

Next extend the group $\langle G, u\rangle$ by the infinite cyclic group $\langle v\rangle$ to get the group $J=\langle G, u, v\rangle$ where the action of $v$ under conjugation is as follows: $v^{-1} a_{i} v=a_{i+1}$, the shift automorphism, and $v^{-1} u v=u^{-1}$. Note that $\langle G, v\rangle=\left\langle a_{0}, v\right\rangle, J=\left\langle a_{0}, u, v\right\rangle$ and $J$ is right orderable.

We now show that $J$ has no discrete right order. Suppose that $c:=g u^{j} v^{k}$ is a minimal positive element under a right order $<$ on $J$. Then $k \neq 0$, since otherwise the restriction of the right order $<$ to $\langle G, u\rangle$ would be discrete with $g u^{j}$ as the minimal positive element. We have seen that this is not possible. Next note that $k$ must be even, otherwise assume without loss of generality that $u>1$. Then $c u c^{-1}=g u^{-1} g^{-1}<1$, which contradicts Lemma 3.2. Since $v u v^{-1}=u^{-1}$, we see that $1<v$ implies $x<v$ for every $x \in\langle G, u\rangle$, in particular $k>0$ and $g u^{j} v^{k-1}>1$. This contradicts the hypothesis that $c$ is the minimal positive element. Similarly we cannot have $1>v$, which finishes the verification that $J$ has no discrete right order.

It is possible for a discretely (right-) ordered group to have a subgroup on which the same order is dense. Indeed, by Corollary 2.2, any densely ordered group is a subgroup of a discretely ordered group, whose order extends the given order. Following is a natural example of this phenomenon for right-ordered groups.

Example 4.4. The Artin braid groups $B_{n}$ have a discrete right-order, which becomes dense when restricted to the commutator subgroup. For each integer $n \geq 2$, $B_{n}$ is the group generated by $\sigma_{1}, \sigma_{2}, \ldots, \sigma_{n-1}$, subject to the relations

$$
\sigma_{i} \sigma_{j}=\sigma_{j} \sigma_{i} \quad \text { if }|i-j|>1 \quad \text { and } \quad \sigma_{i} \sigma_{j} \sigma_{i}=\sigma_{j} \sigma_{i} \sigma_{j} \quad \text { if }|i-j|=1 .
$$

It was shown by Dehornoy (see [Dehornoy 1994] and [Dehornoy et al. 2002]) that each $B_{n}$ is right-orderable (but not orderable, for $n>2$ ). The positive cone consists of all elements expressible as a word in the $\sigma_{i}$ such that the generator with the lowest subscript occurs with only positive exponents. This right-order is discrete, with smallest positive element $\sigma_{n-1}$. On the other hand, it is shown in [Clay and Rolfsen 2007] that the Dehornoy order, when restricted to the commutator subgroup $B_{n}^{\prime}=\left[B_{n}, B_{n}\right]$, is a dense order for $n \geq 3$. For $n=3, B_{n}^{\prime}$ is free (on two generators). For $n \geq 5, B_{n}^{\prime}$ is finitely-generated and perfect [Gorin and Lin 1969], so $B_{n}$ is an example of a nonlocally indicable discretely right-orderable group for $n \geq 5$.

Now consider the braid group $B_{3}$ with its two generators $\sigma_{1}$ and $\sigma_{2}$ and let $H$ be the subgroup generated by $\sigma_{1}^{2}$ and $\sigma_{2}^{2}$. Crisp and Paris [2001] showed that $H$ is a free group with free basis $\sigma_{1}^{2}$ and $\sigma_{2}^{2}$. The Dehornoy order restricted to this 
subgroup has the least positive element $\sigma_{2}^{2}$. This gives an alternative construction of discrete right-orders on a free group.

Bergman [1991] published the first examples of groups which are right-orderable and not locally indicable; some of his examples are finitely generated and perfect. We shall argue that they can be given a discrete right-order.

If $G$ is a group acting on a set and $x_{1}, \ldots, x_{n}$ are elements of the set, then $\operatorname{Stab}_{G}\left(x_{1}, \ldots, x_{n}\right)$ will denote the pointwise stabilizer of $\left\{x_{1}, \ldots, x_{n}\right\}$ in $G$, namely $\left\{g \in G \mid g x_{i}=x_{i}\right.$ for all $\left.i\right\}$. Also $I$ will denote the identity matrix of $\mathrm{SL}_{2}(\mathbb{R})$. We have an action of $\mathrm{SL}_{2}(\mathbb{R})$ on the one point compactification $\overline{\mathbb{R}}=\mathbb{R} \cup\{\infty\} \cong S^{1}$, the circle, given by the rule

$$
\left(\begin{array}{ll}
a & b \\
c & d
\end{array}\right)(x)=\frac{a x+b}{c x+d}
$$

This induces a faithful action of $\mathrm{PSL}_{2}(\mathbb{R})$ on $\overline{\mathbb{R}}$. Let $H$ be a finitely generated subgroup of $\mathrm{SL}_{2}(\mathbb{R})$ containing the center $\{ \pm I\}$ of $\mathrm{SL}_{2}(\mathbb{R})$, and let $\bar{H}$ denote its image in $\mathrm{PSL}_{2}(\mathbb{R})$. Since $\mathbb{R}$ is the universal covering space of $S^{1}$, we can lift the action of $\bar{H}$ on $S^{1}$ to an action of a group $G$ on $\mathbb{R}$ by orientation preserving homeomorphisms. In this situation, $G$ will have a central subgroup $Z \cong \mathbb{Z}$ such that $G / Z \cong \bar{H}$ and $Z$ acts fixed point freely on $\mathbb{R}$. Also if $\pi: \mathbb{R} \rightarrow S^{1}$ is the associated covering map and $p \in \mathbb{R}$, then $\operatorname{Stab}_{\bar{H}}(\pi p)=Z \operatorname{Stab}_{G}(p) / Z \cong \operatorname{Stab}_{G}(p)$.

Proposition 4.6. Let $H$ be a finitely generated subgroup of $\mathrm{SL}_{2}(\mathbb{R})$ with $-I \in H$ and let $G$ be its lift to orientation preserving homeomorphisms of $\mathbb{R}$ (as described above). Suppose $H$ contains a diagonal matrix other than $\pm I$. Then $G$ has a discrete right order.

To prove this, we need an auxiliary result:

Lemma 4.7 [Linnell 2006, Lemma 2.2]. Let $G$ be a right ordered group, let $H$ be a convex subgroup of $G$ and let $<$ be any right order on $H$. Then there exists a right order on $G$ whose restriction to $H$ is $<$, and $H$ is still a convex subgroup under this new right order.

Proof of Proposition 4.6. Let us examine $\operatorname{Stab}_{H}(0)$ and $\operatorname{Stab}_{H}(0, \infty)$ with the action given by (4.5). The former is the lower triangular matrices, that is, the matrices above with $b=0$; we shall denote by $L$ those lower triangular matrices which lie in $H$. The latter is given by the diagonal matrices; we shall denote by $D$ those diagonal matrices which lie in $H$.

Thus we have an action of $H$ on $S^{1}$ and two points $p_{1}, p_{2} \in S^{1}$ such that $\operatorname{Stab}_{H}\left(p_{1}\right)=L$ and $\operatorname{Stab}_{H}\left(p_{1}, p_{2}\right)=D$. Let $p_{3} \in S^{1}$ be distinct from $p_{1}, p_{2}$. Then $\operatorname{Stab}_{H}\left(p_{1}, p_{2}, p_{3}\right)=\{ \pm I\}$. 
Now we can lift the action of $H$ on $S^{1}$ to an action of $G$ on $\mathbb{R}$ by orientation preserving homeomorphisms; $G$ will have a central subgroup $Z \cong \mathbb{Z}$ such that $G / Z \cong H /\{ \pm I\}$. For $i=1,2,3$, let $q_{i} \in \mathbb{R}$ be a lift of $p_{i}$. Then $Q:=$ $\operatorname{Stab}_{G}\left(q_{1}, q_{2}\right) \cong D /\{ \pm I\}$. We now define a right order on $G$ in the usual way when we have a group acting on $\mathbb{R}$. The positive cone is the set of all $g \in G$ such that $g\left(q_{i}\right)>q_{i}$ for the smallest $i$ such that $g\left(q_{i}\right) \neq q_{i}$. This right order will have the property that $Q$ is a (smallest nontrivial) convex subgroup of $G$. If $Q \cong \mathbb{Z}$, then it would follow that the above defined right order will be discrete, but this is not true in general. However since $H$ is finitely generated, $H \subseteq \mathrm{SL}_{2}(R)$ for some finitely generated subring $R$ of $\mathbb{R}$. By [Samuel 1966, Théorème 1], the group of units of a finitely generated integral domain is finitely generated, hence $D$ is also finitely generated. We deduce that $D /\{ \pm I\}$ is a finitely generated free abelian group. Thus $Q$ is also a finitely generated free abelian group and hence has a discrete right order by Corollary 2.2. The result now follows from Lemma 4.7 .

\section{References}

[Baumslag 1968] G. Baumslag, "On the residual nilpotence of certain one-relator groups", Comm. Pure Appl. Math. 21 (1968), 491-506. MR 38 \#3327 Zbl 0186.32101

[Bergman 1991] G. M. Bergman, "Right orderable groups that are not locally indicable", Pacific J. Math. 147:2 (1991), 243-248. MR 92e:20030 Zbl 0677.06007

[Botto Mura and Rhemtulla 1977] R. Botto Mura and A. Rhemtulla, Orderable groups, Lecture Notes in Pure and Applied Mathematics 27, Marcel Dekker, New York, 1977. MR 58 \#10652 Zbl 1071.20508

[Brodskiı̌ 1984] S. D. Brodskiŭ, "Equations over groups, and groups with one defining relation", Sibirsk. Mat. Zh. 25:2 (1984), 84-103. MR 86e:20026 Zbl 0579.20020

[Burns and Hale 1972] R. G. Burns and V. W. D. Hale, "A note on group rings of certain torsion-free groups”, Canad. Math. Bull. 15 (1972), 441-445. MR 46 \#9149 Zbl 0244.16006

[Charney 2007] R. Charney, “An introduction to right-angled Artin groups”, Geom. Dedicata 125 (2007), 141-158. MR 2008f:20076 Zbl 1152.20031

[Clay and Rolfsen 2007] A. Clay and D. Rolfsen, "Densely ordered braid subgroups", J. Knot Theory Ramifications 16:7 (2007), 869-877. MR 2008g:20077 Zbl 1139.20033

[Crisp and Paris 2001] J. Crisp and L. Paris, "The solution to a conjecture of Tits on the subgroup generated by the squares of the generators of an Artin group", Invent. Math. 145:1 (2001), 19-36. MR 2002j:20069 Zbl 1002.20021

[Dehornoy 1994] P. Dehornoy, "Braid groups and left distributive operations", Trans. Amer. Math. Soc. 345:1 (1994), 115-150. MR 95a:08003 Zbl 0837.20048

[Dehornoy et al. 2002] P. Dehornoy, I. Dynnikov, D. Rolfsen, and B. Wiest, Why are braids orderable?, Panoramas et Synthèses [Panoramas and Syntheses] 14, Société Mathématique de France, Paris, 2002. MR 2004e:20062 Zbl 1048.20021

[Duchamp and Krob 1992] G. Duchamp and D. Krob, "The lower central series of the free partially commutative group”, Semigroup Forum 45:3 (1992), 385-394. MR 93e:20047 Zbl 0814.20025 
[Gorin and Lin 1969] E. A. Gorin and V. J. Lin, "Algebraic equations with continuous coefficients and some problems of the algebraic theory of braids", Mat. Sb. (N.S.) 78 (1969), 579-610. In Russian; translated in Math. USSR, Sb. 7 (1969), 569-596. MR 40 \#4939 Zbl 0211.54905

[Linnell 2001] P. A. Linnell, "Left ordered groups with no non-abelian free subgroups", J. Group Theory 4:2 (2001), 153-168. MR 2002h:06018 Zbl 0982.06013

[Linnell 2006] P. A. Linnell, "The topology on the space of left orderings of a group", preprint, 2006, Available at http://arxiv.org/abs/math/0607470. arXiv math/0607470v2

[Longobardi et al. 2000] P. Longobardi, M. Maj, and A. Rhemtulla, "When is a right orderable group locally indicable?", Proc. Amer. Math. Soc. 128 (2000), 637-641. MR 2000j:20072 Zbl 0941.20037

[Morris 2006] D. W. Morris, "Amenable groups that act on the line", Algebr. Geom. Topol. 6 (2006), 2509-2518. MR 2008c:20078 Zbl pre05118602

[Samuel 1966] P. Samuel, “À propos du théorème des unités”, Bull. Sci. Math. (2) 90 (1966), 89-96. MR 34 \#4296 Zbl 0166.30701

Communicated by Efim Zelmanov

Received 2008-08-19 Revised 2009-03-09 Accepted 2009-04-17

linnell@math.vt.edu Department of Mathematics, Virginia Tech, Blacksburg, VA 24061-0123, United States http://www.math.vt.edu/people/plinnell/

akbar@math.ualberta.ca

Department of Mathematical \& Statistical Sciences, University of Alberta, Edmonton, AL T6G2G1, Canada http://www.math.ualberta.ca/Rhemtulla_A.html

rolfsen@math.ubc.ca

University of British Columbia, Mathematics Department, 1984 Mathematics Road, Vancouver, BC V6T 1Z2, Canada http://www.math.ubc.ca/ rolfsen/ 